\title{
Do not forget leishmaniasis
}

\section{Editorial}

Leishmaniasis is a public health problem. Co-infection with human immunodeficiency virus (HIV) and global warming have contributed to its expansion. The problem of the Mediterranean basin peaked in the 90 s and through multidrug therapy (HAART) used against AIDS, the numbers are in decline. ${ }^{1}$ Remain the problem of kennels with infected animals in the UK and US, which may open the road to drive new vectors such as ticks, or mechanical infections through blood transfusion.

As leishmaniasis is a vector-borne disease, the condition from the point of view of the Leishmania parasite, is highly efficient; thus, becoming a model of infection with clinical, parasitological and immunological manifestations, ideal for understanding the relationship between a pathogen and its mammalian host. It also has the advantage of being able to reproduce the clinical manifestations seen in humans in syngeneic strains of mice. From the point of view of the vectors, sandflies have a worldwide distribution in tropical and subtropical areas. Both genera Phlebotomus and Lutzomyia are recognized as vectors in the transmission of leishmaniasis.

It is important to note that female mosquitoes require a blood intake to mature their eggs, leading to evolutionary genetic changes that help spread of leishmaniasis. Thus, the saliva is rich in phlebotomine substances promoting blood intake. One of these substances is maxadilan, a neuropeptide similar to calcitonin gene related peptide (CGRP) that Leishmania parasites used in their favor to infect more efficiently. ${ }^{2}$

In Brazil, the group led by Manoel Barral-Netto and Aldina Barral from FioCruz Bahia, has been working on modifying the immune response using sandfly saliva during the course of the disease. They had shown that Lutzomyia longipalpis saliva is protective against infection, but saliva from Lutzomyia intermedia favors neutrophils and monocytes recruitment, which are palatable, cells to be invaded by the parasite. ${ }^{3}$

The high density and variability of Leishmania and sandflies, in addition to climate change and urbanization of phlebotomines, remind us how far we are from effective solutions to combat this disease.

Our group has demonstrated the participation of epidermal dendritic cells in both human and murine cutaneous leishmaniasis, and have shown that most of the tissue-damage observed in tegumentary leishmaniasis is caused by the immune response a not by the parasite. This immunopathology is promoted by inadequate epidermal signals driven by dendritic cells. We have also demonstrated increased numbers of IL-10+ Langerhans cells in lesions of patients with intermediate cutaneous leishmaniasis as compare with localized cutaneous leishmaniasis, and have shown that the adoptive transfer of splenic dendritic cells confers protection to Leishmania-infection in a neonatal BALB/c model of the disease., ${ }^{4,5}$

We have also studied the antimicrobial peptide LL-37, a bioactive peptide derived from the human cathelicidin CAP18, predominately expressed on epithelial tissue during inflammation. ${ }^{6}$ Besides its antibacterial function, LL-37 has a role as an inflammatory mediator that has not been studied in leishmaniasis. We evaluated the
Volume I Issue 3 - 2014

\author{
Felix J Tapia \\ Laboratory of Molecular Biology, Central University of \\ Venezuela, Venezuela \\ Correspondence: Felix J Tapia, Laboratory of Molecular \\ Biology, Institute of Biomedicine, Central University \\ of Venezuela, Apartado 4043, Caracas, Venezuela, Tel \\ 582128625326; Email felix.tapia@gmail.com \\ Received: July 15, 2014 | Published: July 16, 2014
}

leishmanicidal activity of LL-37, its effect on the infection with $L$. mexicana promastigotes and murine bone marrow derived dendritic cells, and the lymphoproliferative response against the parasite. The results suggest that LL-37 is a multifunctional regulator of the innate and adaptive immune response against $L$. mexicana, having a leishmanicidal activity, increasing phagocytosis on dendritic cells and macrophages, and depending on the concentration acting as a cytokine, activating or suppressing the adaptive immune response. ${ }^{6}$

In order to understand the immunoregulatory mechanisms that are activated after the resolution of skin lesions in leishmaniasis, we have used a single homotypic stressor just before the inoculation of Leishmania parasites in susceptible and resistant mouse models of L. mexicana infection. The stressor consisted of immobilization by placement, for 2 or 8 hours, in restraining cages. The results suggest that stress by immobilization may modulate the genetically predetermined immune response to Leishmania parasite. In addition and perhaps more significant, it indicates that animals manage the same acute and homotypic stress in a different manner, some exacerbating their lesions and others by healing by their genetic legacy. ${ }^{7,8}$

All together these results are helping us in understanding the mechanisms behind the immunopathogenesis in leishmaniasis. At present, we are concentrated in characterizing the different subtypes of dendritic cells, innate lymphoid cells and the so-called guardians of the immune response.

\section{Acknowledgments}

None.

\section{Conflicts of interest}

Authors declare that there is no conflict of interest.

\section{References}

1. Alvar J, Aparicio P, Aseffa A, et al. The relationship between leishmaniasis and AIDS: the second 10 years. Clin Microbiol Rev. 2008;21(2):334-359.

2. Lerner EA, Ribeiro JM, Nelson RJ, et al. Isolation of maxadilan, a potent vasodilatory peptide from the salivary glands of the sand fly Lutzomyia longipalpis. J Biol Chem. 1991;266(17):11234-11236.

3. Tavares NM, Silva RA, Costa DJ, et al. Lutzomyia longipalpis saliva or salivary protein LJM19 protects against Leishmania braziliensis and the saliva of its vector, Lutzomyia intermedia. PLoS Negl Trop Dis. 2011;5(5):e1169. 
4. Tapia FJ, Caceres-Dittmar G, Sanchez MA. Inadequate epiderma homing leads to tissue damage in human cutaneous leishmaniasis. Immunol Today. 1994;15(4):160-165.

5. Ponce LV, Corado J, Diaz NL, et al. Adoptive transfer of dendritic cells modulates immunogenesis and tolerogenesis in a neonatal model of murine cutaneous leishmaniasis. Kinetoplastid Biol Dis. 2005;4(1):2.

6. Tapia FJ, Diaz NL, Rodriguez OI, et al. Tegumentary leishmaniasis: immunology and molecular biology. Gaz med Bahia. 2009;79(Suppl 3):84-90.
7. Ruiz MR, Quinones AG, Diaz NL, et al. Acute immobilization stress induces clinical and neuroimmunological alterations in experimental murine cutaneous leishmaniasis. Br J Dermatol. 2003;149(4):731-738.

8. Aguilar E, Diaz NL, Sanchez MA, et al. Patron de citocinas en higado y bazo de perros con leishmaniasis visceral natural. Rev Fac Cienc Vet. 2009;50(2):89-95 